\title{
State of Charge Estimation Based on Microscopic Driving Parameters for Electric Vehicle's Battery
}

\author{
Enjian Yao, 1,2 Meiying Wang, ${ }^{1,2}$ Yuanyuan Song, ${ }^{2}$ and Yang Yang ${ }^{2}$ \\ ${ }^{1}$ MOE Key Laboratory for Urban Transportation Complex Systems Theory and Technology, Beijing Jiaotong University, \\ Beijing 100044, China \\ ${ }^{2}$ School of Traffic and Transportation, Beijing Jiaotong University, Haidian District, Beijing 100044, China
}

Correspondence should be addressed to Meiying Wang; 12120914@bjtu.edu.cn

Received 27 September 2013; Revised 17 November 2013; Accepted 25 November 2013

Academic Editor: Wuhong Wang

Copyright (C) 2013 Enjian Yao et al. This is an open access article distributed under the Creative Commons Attribution License, which permits unrestricted use, distribution, and reproduction in any medium, provided the original work is properly cited.

\begin{abstract}
Recently, battery-powered electric vehicle (EV) has received wide attention due to less pollution during use, low noise, and high energy efficiency and is highly expected to improve urban air quality and then mitigate energy and environmental pressure. However, the widespread use of EV is still hindered by limited battery capacity and relatively short cruising range. This paper aims to propose a state of charge (SOC) estimation method for EV's battery necessary for route planning and dynamic route guidance, which can help EV drivers to search for the optimal energy-efficient routes and to reduce the risk of running out of electricity before arriving at the destination or charging station. Firstly, by analyzing the variation characteristics of power consumption rate with initial SOC and microscopic driving parameters (instantaneous speed and acceleration), a set of energy consumption rate models are established according to different operation modes. Then, the SOC estimation model is proposed based on the presented EV power consumption model. Finally, by comparing the estimated SOC with the measured SOC, the proposed SOC estimation method is proved to be highly accurate and effective, which can be well used in EV route planning and navigation systems.
\end{abstract}

\section{Introduction}

In recent years, energy and environment have suffered from heavy pressure caused by rapidly increased gasoline and diesel powered vehicles, and the growing concern about energy reserves and environmental quality of cities has called for sustainable transportation technologies and motivated active research on vehicles with alternative energy sources $[1,2]$. The electric vehicles (EVs), which run on electricity, have received wide attention due to less pollution during use, low noise, and high energy efficiency $[3,4]$.

Although EV has considerable advantages, the popularization of EV is still hindered by limited battery capacity and relatively short cruising range. Up to now, the battery management system (BMS) has been developed as one of the EV's key technologies, and the accurate estimation of SOC of battery is viewed as a critical part of BMS. Since the battery is a strong nonlinear and time-variability system for its complicated electrochemical process $[5,6]$, the SOC is affected by many factors such as open-circuit voltage, self-rescue effect, temperature, charge and discharge efficiency, and circle life. Although a great many SOC estimation methods have been explored by researchers, the accurate estimation of SOC is very difficult and complex due to limited battery models and parametric uncertainties [7].

According to different methodologies, the SOC estimation methods can be classified into four categories, including direct measurement, book-keeping estimation, adaptive system, and hybrid methods [8]. The direct measurement methods refer to measuring the SOC based on the battery properties (battery voltage, battery impedance, etc.), which mainly include open-circuit voltage (OCV) method $[9,10]$, terminal voltage method [11], impedance measurement method [12, $13]$, and impedance spectroscopy method $[14,15]$. The bookkeeping is a method based on current measurement and integration, which uses battery discharging current data and other relevant data (self-discharge rate of battery, temperature, charge/discharge efficiency, etc.) as inputs [16]. The two 
main book-keeping estimation methods are Coulomb counting method and modified Coulomb counting method [17]. With the development of artificial intelligence, various new adaptive systems for SOC estimation have been explored, which mainly include back propagation (BP) neural network $[18,19]$, radial basis function (RBF) neural network [20], fuzzy logic methods [21, 22], support vector machine [23], fuzzy neural network [24], and Kalman filter [25, 26]. In addition, the hybrid methods are explored by some researchers, which take advantage of multiple SOC estimation methods to improve the estimation accuracy [27].

Although extensive research has been undertaken in exploring approaches to estimate the SOC of battery based on the complex external characteristic of battery as mentioned above, few papers have been devoted to studying the relationship between the SOC of EV's battery and microscopic driving parameters. This means that the SOC at some specific points, which is necessary for EV route planning, cannot be predicted before the travel using the current estimation methods. Therefore, in an attempt to overcome the limitations of current SOC estimation methods for EV's battery, this paper proposes a SOC estimation approach based on the forecasted microscopic driving parameters, which can predict the power consumption and SOC accurately before departure and then reduce the risk of running out of electricity before arriving at the destination or charging station.

This paper is organized into four sections. In the first section, the background and significance on SOC estimation models are provided, along with a review of existing studies on SOC estimation. In the second section, by analyzing the impacts of initial SOC for each second and microscopic driving parameters on power consumption rate, the EV power consumption rate models for different operation modes are established. On that basis, the SOC estimation model is proposed, which uses the initial SOC, instantaneous speed, and acceleration as input variables. In the third section, the model parameters are calibrated and the estimation results are discussed by comparing the measured SOC with estimated SOC. The conclusions and future work are then provided in the last section.

\section{Modeling}

Since the SOC of battery is decided by the initial SOC and power consumption during the discharge time, in order to establish the relationship between SOC and microscopic driving parameters, it is important to study the impact of microscopic driving parameters on power consumption of $\mathrm{EV}$. Therefore, in this paper, the EV power consumption rate models for different operation modes are set up firstly, which give a full consideration to the influence of the initial SOC for each second, instantaneous speed, and acceleration on the power consumption rate. Then, the SOC estimation model is proposed based on the presented EV power consumption model.

2.1. Data Source. The data used for establishing and verifying the power consumption rate models and SOC estimation

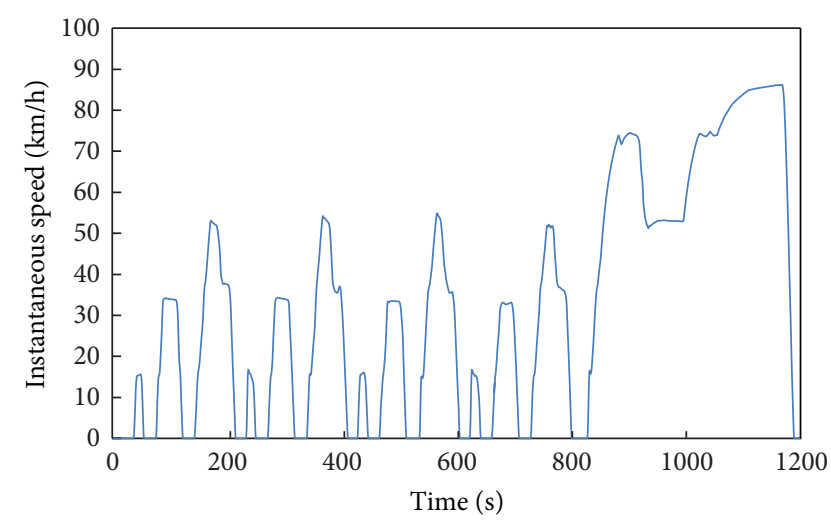

Figure 1: New European Driving Cycle.

TABLE 1: Correlation coefficient.

\begin{tabular}{lccc}
\hline $\begin{array}{l}\text { Correlation } \\
\text { coefficient }\end{array}$ & $\begin{array}{c}\text { Initial SOC for } \\
\text { each second }(\%)\end{array}$ & $\begin{array}{c}\text { Speed } \\
(\mathrm{km} / \mathrm{h})\end{array}$ & $\begin{array}{c}\text { Acceleration } \\
\left(\mathrm{m} / \mathrm{s}^{2}\right)\end{array}$ \\
\hline $\begin{array}{l}\text { Power consumption } \\
\text { rate }\end{array}$ & -0.136 & 0.765 & 0.410 \\
\hline
\end{tabular}

model are collected by a chassis dynamometer test with New European Driving Cycle (NEDC). NEDC is a driving cycle consisting of four repeated ECE-15 driving cycles and an Extra-Urban Driving Cycle (EUDC), as shown in Figure 1, and NEDC is supposed to represent the typical usage of a car in China and Europe, and designed to assess the emission levels of car engines and fuel economy in passenger cars (excluding light trucks and commercial vehicles). The data include four NEDCs and contain time, vehicle speed, battery working current, battery voltage, and so forth. In the paper, the data of the first three NEDCs are used to set up the EV power consumption rate models and SOC estimation model, and the data of the rest one driving cycle are used to verify the accuracy of the proposed models.

2.2. Model Methodology. Both the initial SOC for each second and microscopic driving parameters have a significant impact on the power consumption rate. Therefore, in this section, by analyzing the correlation between the power consumption rate and initial SOC for each second and microscopic driving parameters, the EV power consumption rate models are built up according to different operation modes. On that basis, the SOC estimation model is established.

2.2.1. Modeling Power Consumption Rate for EVs. The correlation coefficients between the EV power consumption rate and initial SOC for each second, instantaneous speed, and acceleration are summarized in Table 1 . It can be concluded that there is a relatively strong correlation between power consumption rate and these parameters.

Further, the variation characteristics of power consumption rate with these parameters are analyzed (shown in Figure 2). According to Figure 2(a), the change rules of power consumption rate vary in different initial SOC for 


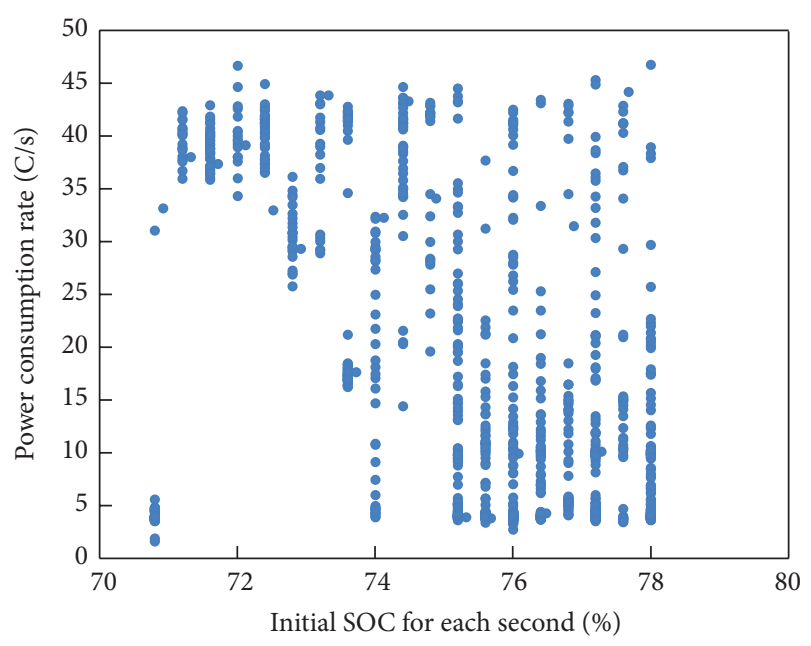

(a)

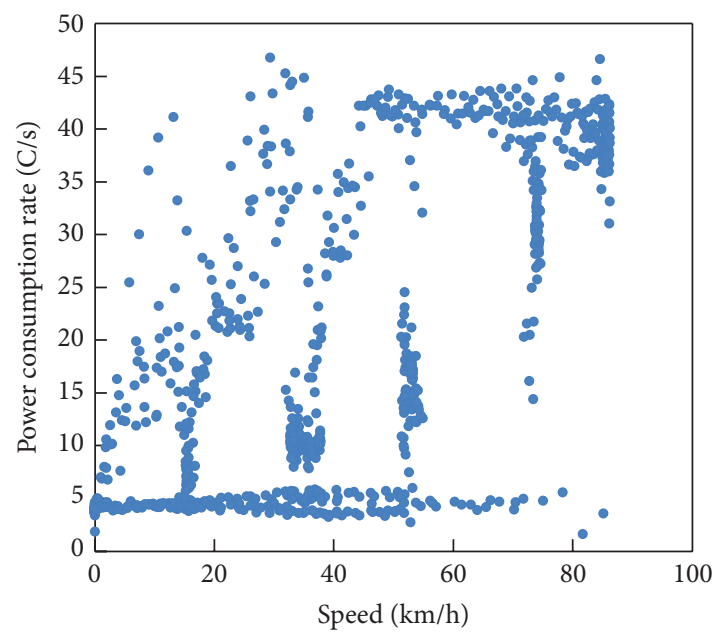

(b)

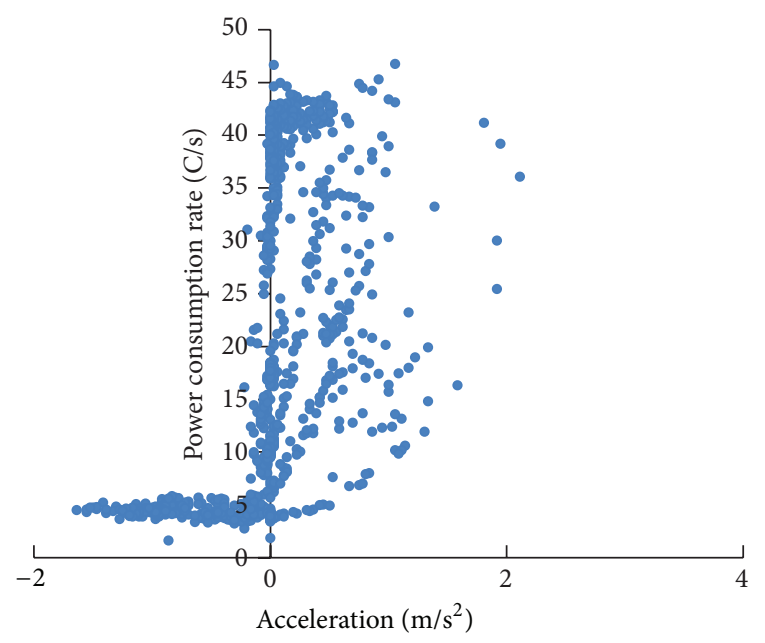

(c)

FIGURE 2: Variation characteristics of power consumption rate with initial SOC for each second and microscopic driving parameters. each second. Figures 2(b) and 2(c) illustrate graphically the nonlinear relationship between power consumption rate and microscopic driving parameters. It is clear that power consumption rate generally tends to increase with the increase of speed and acceleration. In addition, the gradient of the power consumption rate for decelerating mode is generally smaller than that for accelerating mode, in which it should be noted that the power consumption rate varies in different operation modes. Therefore, in this paper, the running status of EVs is divided into four operation modes, including accelerating, decelerating, cruising, and idling, and then four power consumption rate models are established, respectively.

Based on the analysis above, this paper attempts to establish the EV power consumption rate models for different operation modes that require initial SOC for each second, instantaneous speed and acceleration as independent variables.

The derivation of the model structure involves experimentation with numerous polynomial combinations of the initial SOC for each second, speed, and acceleration. Based on the VT-Micro model of fuel consumption and emission for gasoline and diesel powered vehicles [28, 29], the final regression model includes the linear initial SOC for each second coupled with a combination of linear, quadratic, and cubic speed and acceleration terms because it indicates a relatively high goodness of fit for the collected data as (1). Since the above model may produce negative dependent variable values in a few instances, a data transformation method [29] is applied to the model presented in (1), and the exponential function model presented in (2) is obtained. Furthermore, the power consumption rate for idling mode is estimated using the average value of the power consumption of every second:

$$
q= \begin{cases}a_{0} \times S+\sum_{i=0}^{3} \sum_{j=0}^{3}\left(a_{i, j} \times v^{i} \times a^{j}\right), & a>0 \\ b_{0} \times S+\sum_{i=0}^{3} \sum_{j=0}^{3}\left(b_{i, j} \times v^{i} \times a^{j}\right), & a<0 \\ c_{0} \times S+\sum_{i=0}^{3}\left(c_{i} \times v^{i}\right), & a=0, v \neq 0 \\ \bar{q}, & a=0, v=0, \\ \exp \left(l_{0} \times S+\sum_{i=0}^{3} \sum_{j=0}^{3}\left(l_{i, j} \times v^{i} \times a^{j}\right)\right) & a>0 \\ \exp \left(m_{0} \times S+\sum_{i=0}^{3} \sum_{j=0}^{3}\left(m_{i, j} \times v^{i} \times a^{j}\right)\right) & a<0 \\ \exp \left(n_{0} \times S+\sum_{i=0}^{3}\left(n_{i} \times v^{i}\right)\right) & a=0, v \neq 0\end{cases}
$$

where $q$ is the power consumption rate, namely, the power consumption of each second, $\mathrm{C} / \mathrm{s} ; \mathrm{S}$ is the initial SOC for each second, $\% ;\left(a_{0}, a_{i, j}\right),\left(b_{0}, b_{i, j}\right)$, and $\left(c_{0}, c_{i}\right)$ are the coefficients of accelerating, decelerating, and cruising, respectively, 
TABLE 2: Results of parameters calibration for power consumption rate models.

\begin{tabular}{|c|c|c|c|c|}
\hline Variable & $\begin{array}{c}\text { Accelerating } \\
\text { coefficients ( } t \text {-value) }\end{array}$ & $\begin{array}{c}\text { Decelerating } \\
\text { coefficients }(t \text {-value) }\end{array}$ & $\begin{array}{c}\text { Cruising } \\
\text { coefficients }(t \text {-value })\end{array}$ & $\begin{array}{c}\text { Idling } \\
\text { coefficients }\end{array}$ \\
\hline Constant & $1.862(14.816)$ & $1.098(18.314)$ & $0.602(2.704)$ & 3.62 \\
\hline$S$ & $0.226(2.132)$ & $0.317(4.743)$ & $0.633(3.002)$ & - \\
\hline$v$ & - & $0.025(42.068)$ & $0.037(10.492)$ & - \\
\hline$v^{2}$ & - & - & - & - \\
\hline$v^{3}$ & - & $1.184 E-006(12.008)$ & $-1.062 E-006(-3.314)$ & - \\
\hline$a$ & - & - & - & - \\
\hline$a^{2}$ & - & - & - & - \\
\hline$a^{3}$ & - & - & - & - \\
\hline$v a$ & $0.098(23.0313)$ & 0.115 (49.117) & - & - \\
\hline$v a^{2}$ & - & $0.152(35.344)$ & - & - \\
\hline$v a^{3}$ & $-0.030(-6.753)$ & $0.053(28.475)$ & - & - \\
\hline$v^{2} a$ & - & - & - & - \\
\hline$v^{2} a^{2}$ & - & - & - & - \\
\hline$v^{2} a^{3}$ & - & - & - & - \\
\hline$v^{3} a$ & - & - & - & - \\
\hline$v^{3} a^{2}$ & $-5.054 E-005(-13.667)$ & $-2.324 E-006(-13.310)$ & - & - \\
\hline$v^{3} a^{3}$ & $2.965 E-005(12.637)$ & - & - & - \\
\hline Adjusted $R^{2}$ & 0.947 & 0.894 & 0.927 & - \\
\hline
\end{tabular}

$v$ is the instantaneous vehicle speed, $\mathrm{km} / \mathrm{h} ; a$ is the acceleration, $\mathrm{m} / \mathrm{s}^{2} ; S$ is the initial SOC for each second, \%; $R^{2}$ is the coefficient of determination.

for polynomial model, $i, j=0,1,2,3 ;\left(l_{0}, l_{i, j}\right),\left(m_{0}, m_{i, j}\right)$, and $\left(n_{0}, n_{i}\right)$ are the coefficients of accelerating, decelerating, and cruising respectively, for exponential model, $i, j=$ $0,1,2,3 ; v$ is the instantaneous speed of $\mathrm{EV}, \mathrm{km} / \mathrm{h} ; a$ is the instantaneous acceleration, $\mathrm{m} / \mathrm{s}^{2} ; \bar{q}$ is the average power consumption of every second for idling, $\mathrm{C} / \mathrm{s}$.

2.2.2. Estimating SOC for EVs. Based on the power consumption rate models proposed above, the power consumption during the discharge time can be obtained by the time integral as

$$
\mathrm{Q}=\sum_{i=1}^{n} q_{i} \times t
$$

where $Q$ is the power consumption during the discharge time, $\mathrm{C} ; n$ is the discharge time, $s ; q_{i}$ is the power consumption of each second, namely, the power consumption rate, $\mathrm{C} / \mathrm{s} ; t$ is the time interval, $1 \mathrm{~s}$.

Thus, the SOC estimation model based on the initial SOC and microscopic driving parameters can be calculated by

$$
S_{n+1}=f\left(S_{0}, v, a\right)=S_{0}-\frac{Q}{3600 \times Q_{r}}=S_{0}-\frac{\sum_{i=0}^{n} q_{i} \times t}{3600 \times Q_{r}},
$$

where $S_{n+1}$ is state of charge after the discharge time $n, \% ; S_{0}$ is the initial SOC, $\% ; Q_{r}$ is the nominal capacity of battery given by the manufacturer, Ah.

\section{Results and Analysis}

In this section, using SPSS software, the parameters of $\mathrm{EV}$ power consumption rate models for different operation modes are calibrated, respectively, based on regression analysis method. Further, the estimated power consumption and SOC are compared with the measured power consumption and SOC to verify the accuracy of the proposed EV power consumption rate estimation models and the SOC estimation model.

3.1. Parameter Calibration. Based on the data of the first three driving cycles mentioned above, using regression analysis method, the parameters for accelerating, decelerating, cruising, and idling are calibrated, respectively. Since the use of polynomial speed and acceleration terms may result in multicollinearity between the independent variables due to the dependency of the variables, stepwise regression analysis method is utilized in the parameters calibration. However, the study shows that heteroskedasticity exists in the accelerating model calculated with ordinary least square (OLS). Therefore, for accelerating mode, weighted least square (WLS) is used for model calibration. The results of parameters calibration for power consumption rate models are shown in Table 2.

According to Table 2, the statistical results indicate a relatively high goodness of fit for power consumption rate estimation (the adjusted $R^{2}$ values in excess of 0.89 and the absolute $t$-values of the variables in excess of 1.96 for all the operation modes).

3.2. Estimation Results. On the one hand, in order to evaluate the accuracy of the proposed power consumption rate estimation models, the measured power consumption rate data are compared with the regression models estimation through 
TABLE 3: Results of MAPE for different operation modes.

Operation modes Accelerating Decelerating Cruising Idling

\begin{tabular}{lllll}
\hline MAPE & $8.74 \%$ & $9.92 \%$ & $9.45 \%$ & $9.67 \%$ \\
\hline
\end{tabular}

calculating the mean absolute percentage error (MAPE). MAPE is calculated by

$$
\text { MAPE }=\frac{1}{n} \sum_{i=1}^{n}\left|\frac{Q_{i}-Q_{i}^{\prime}}{Q_{i}}\right| \times 100 \%,
$$

where $Q_{i}$ is the measured power consumption, $C ; Q_{i}^{\prime}$ is the estimated power consumption using the proposed models, $\mathrm{C}$; $n$ is the number of tested data samples.

With the purpose of ensuring consistency in comparison, in this paper, the data of the rest one driving cycle mentioned above are utilized to verify the accuracy of proposed models. The MAPEs for second-by-second power consumption illustrate that the models are relatively accurate with MAPE ranging from $8.74 \%$ to $9.92 \%$ (shown in Table 3).

Further, in order to evaluate the applicability of the proposed models in EV route planning and navigation systems, the MAPE of a specific route is also taken as evaluation indicator. The rest one driving cycle is divided into different time intervals ( $2 \mathrm{~min}, 4 \mathrm{~min}, 6 \mathrm{~min}, 8 \mathrm{~min}, 10 \mathrm{~min}, 12 \mathrm{~min}, 14 \mathrm{~min}$, $16 \mathrm{~min}, 18 \mathrm{~min}$, and $20 \mathrm{~min}$ ) and each time interval is regarded as a specific planned route of EV. The power consumption for each time interval is estimated using the proposed models, and then the MAPEs for the planned route are calculated under different time intervals (shown in Figure 3). It is clear that the MAPEs for different time intervals are all less than $4.50 \%$ and the MAPE generally tends to decrease with the increase of length of time interval. Therefore, for the data of NEDC used in this paper, the presented power consumption rate models for different operation modes can estimate the EV power consumption with sufficient accuracy.

On the other hand, the accuracy of the presented SOC estimation model is analyzed and discussed. Due to the limited battery capacity and relatively short cruising range, it is necessary to obtain several specific SOC values of EV's battery during the route planning. Therefore, the several specific SOC values are calculated using the above SOC estimation model. To evaluate the accuracy and applicability of the proposed model in EV route planning and navigation systems, the estimated SOC is compared with the measured SOC (shown in Figure 4). The relative errors for different time points between the measured SOC and estimated SOC are all quite small. Therefore, for the data of NEDC used in the paper, the presented model is appropriate for estimating the SOC with sufficient accuracy and can be well applied in EV route planning and navigation systems.

\section{Conclusions}

The paper presents a novel SOC estimation approach based on the data collected by a chassis dynamometer test with NEDC, which is characterized by fully considering the impacts of microscopic driving parameters on SOC. Firstly,

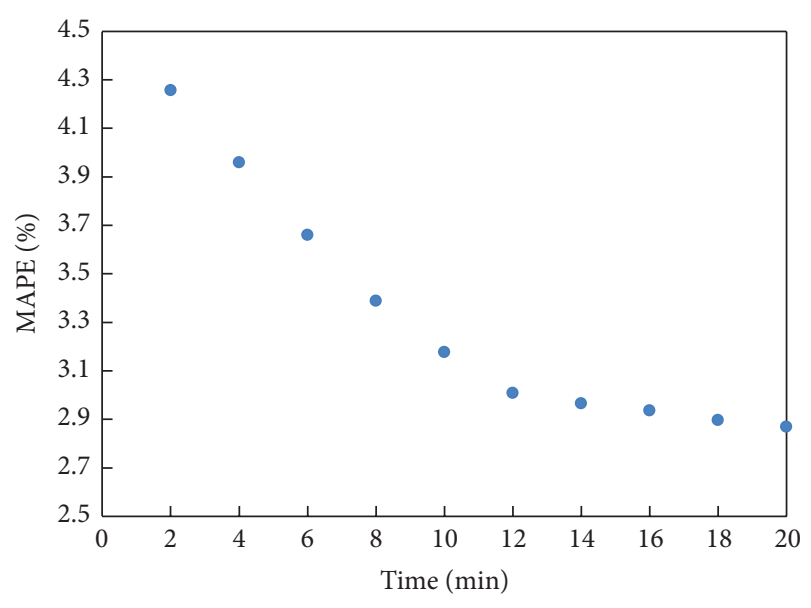

FIGURE 3: MAPEs of power consumption rate model under different time intervals.

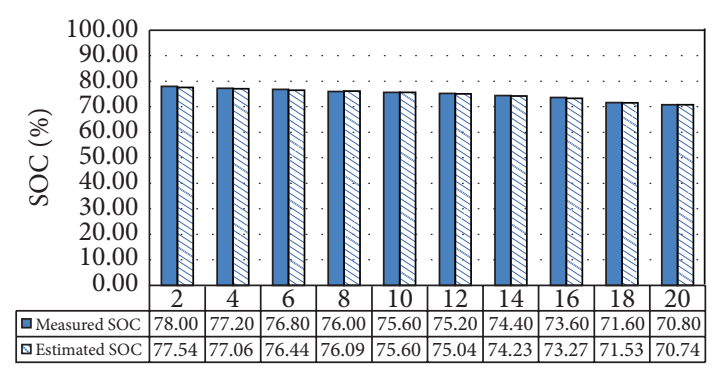

FIGURE 4: Comparison between measured SOC and estimated SOC.

through the variation characteristics analysis of power consumption rate with initial SOC for each second, instantaneous speed, and acceleration, a set of EV power consumption rate models with exponential function are established for different operation modes. Further, using the presented EV power consumption rate models, the SOC estimation model that requires the initial SOC and microscopic driving parameters as input variables is set up. Then, the parameters of EV power consumption rate models for different operation modes are calibrated, respectively, using the regression analysis method. For the data of NEDC used in the paper, the power consumption rate models for different operation modes are relatively accurate with MAPE ranging from $8.74 \%$ to $9.92 \%$, and the SOC estimation model can be well applied in EV route planning and navigation systems to estimate the SOC of battery. In the future practical application, the models presented in this paper can be utilized in conjunction with microscopic traffic simulation technologies or GPS to further demonstrate their application to routing planning and dynamic route guidance for $\mathrm{EV}$.

\section{Conflict of Interests}

The authors declare that there is no conflict of interests regarding the publication of this paper. 


\section{Acknowledgments}

This research is supported by the National 973 Program of China (no. 2012CB725403) and the Research Fund for the Doctoral Program of Higher Education of China (no. 20130009110002).

\section{References}

[1] J. A. Calvo, C. Álvarez-Caldas, J. L. San Román, and P. Cobo, "Influence of vehicle driving parameters on the noise caused by passenger cars in urban traffic," Transportation Research D, vol. 17, no. 7, pp. 509-513, 2012.

[2] H. S. Hamut, I. Dincer, and G. F. Naterer, "Performance assessment of thermal management systems for electric and hybrid electric vehicles," International Journal of Energy Research, vol. 37, no. 1, pp. 1-12, 2013.

[3] L. He, W. Chen, and G. Conzelmann, "Impact of vehicle usage on consumer choice of hybrid electric vehicles," Transportation Research D, vol. 17, no. 3, pp. 208-214, 2012.

[4] Z. P. Wang, P. Liu, J. Cui, Y. Xi, and L. Zhang, "Research on quantitative models of electric vehicle charging stations based on principle of energy equivalence," Mathematical Problems in Engineering, vol. 2013, Article ID 959065, 10 pages, 2013.

[5] H. He, R. Xiong, and J. Fan, "Evaluation of lithium-ion battery equivalent circuit models for state of charge estimation by an experimental approach," Energies, vol. 4, no. 4, pp. 582-598, 2011.

[6] R. T. Doucette and M. D. McCulloch, "Modeling the prospects of plug-in hybrid electric vehicles to reduce $\mathrm{CO}_{2}$ emissions," Applied Energy, vol. 88, no. 7, pp. 2315-2323, 2011.

[7] A. A. A. Elgammal and A. M. Sharaf, "Self-regulating ppaper swarm optimized controller for (photovoltaic-fuel cell) battery charging of hybrid electric vehicles," IET Electrical Systems in Transportation, vol. 2, no. 2, pp. 77-89, 2012.

[8] W. Y. Chang, "The state of charge estimating methods for battery: a review," ISRN Applied Mathematics, vol. 2013, Article ID 953792, 7 pages, 2013.

[9] S. Lee, J. Kim, J. Lee, and B. H. Cho, "State-of-charge and capacity estimation of lithium-ion battery using a new opencircuit voltage versus state-of-charge," Journal of Power Sources, vol. 185, no. 2, pp. 1367-1373, 2008.

[10] S. Abu-Sharkh and D. Doerffel, "Rapid test and non-linear model characterisation of solid-state lithium-ion batteries," Journal of Power Sources, vol. 130, no. 1-2, pp. 266-274, 2004.

[11] S. Sato and A. Kawamura, "A new estimation method of state of charge using terminal voltage and internal resistance for lead acid battery," in Proceedings of the Power Conversion Conference, pp. 565-570, Osaka, Japan, April 2002.

[12] S. Rodrigues, N. Munichandraiah, and A. K. Shukla, "Review of state-of-charge indication of batteries by means of A.C. impedance measurements," Journal of Power Sources, vol. 87, no. 1-2, pp. 12-20, 2000.

[13] F. Huet, "A review of impedance measurements for determination of the state-of-charge or state-of-health of secondary batteries," Journal of Power Sources, vol. 70, no. 1, pp. 59-69, 1998.

[14] R. Li, J. Wu, H. Wang, and G. Li, "Prediction of state of charge of lithium-ion rechargeable battery with electrochemical impedance spectroscopy theory," in Proceedings of the 5th IEEE Conference on Industrial Electronics and Applications (ICIEA '10), pp. 684-688, Taichung, Taiwan, June 2010.
[15] K. Bundy, M. Karlsson, G. Lindbergh, and A. Lundqvist, "An electrochemical impedance spectroscopy method for prediction of the state of charge of a nickel-metal hydride battery at open circuit and during discharge," Journal of Power Sources, vol. 72, no. 2, pp. 118-125, 1998.

[16] H. J. Bergveld, W. S. Kruijt, and P. H. L. Notten, Battery Management Systems, Design By Modeling, vol. 1 of Philips research book series, Kluwer Academic, Boston, Mass, USA, 2002.

[17] K. S. Ng, C.-S. Moo, Y.-P. Chen, and Y.-C. Hsieh, "Enhanced coulomb counting method for estimating state-of-charge and state-of-health of lithium-ion batteries," Applied Energy, vol. 86, no. 9, pp. 1506-1511, 2009.

[18] W. Y. Chang, "State of charge estimation for $\mathrm{LiFePO}_{4}$ battery using artificial neural network," International Review of Electrical Engineering, vol. 7, no. 5, pp. 5874-5800, 2012.

[19] T. Weigert, Q. Tian, and K. Lian, "State-of-charge prediction of batteries and battery-supercapacitor hybrids using artificial neural networks," Journal of Power Sources, vol. 196, no. 8, pp. 4061-4066, 2011.

[20] G. Hongyu, J. Jiuchun, and W. Zhanguo, "Estimating the state of charge for Ni-MH battery in HEV by RBF neural network," in Proceedings of the International Workshop on Intelligent Systems and Applications (ISA '09), pp. 1-4, Wuhan, China, May 2009.

[21] P. Singh, R. Vinjamuri, X. Wang, and D. Reisner, "Design and implementation of a fuzzy logic-based state-of-charge meter for Li-ion batteries used in portable defibrillators," Journal of Power Sources, vol. 162, no. 2, pp. 829-836, 2006.

[22] S. Malkhandi, "Fuzzy logic-based learning system and estimation of state-of-charge of lead-acid battery," Engineering Applications of Artificial Intelligence, vol. 19, no. 5, pp. 479-485, 2006.

[23] T. Hansen and C.-J. Wang, "Support vector based battery state of charge estimator," Journal of Power Sources, vol. 141, no. 2, pp. 351-358, 2005.

[24] Y.-S. Lee, W.-Y. Wang, and T.-Y. Kuo, "Soft computing for battery state-of-charge (BSOC) estimation in battery string systems," IEEE Transactions on Industrial Electronics, vol. 55, no. 1, pp. 229-239, 2008.

[25] L. Xu, J. Wang, and Q. Chen, "Kalman filtering state of charge estimation for battery management system based on a stochastic fuzzy neural network battery model," Energy Conversion and Management, vol. 53, no. 1, pp. 33-39, 2012.

[26] F. Sun, X. Hu, Y. Zou, and S. Li, "Adaptive unscented Kalman filtering for state of charge estimation of a lithium-ion battery for electric vehicles," Energy, vol. 36, no. 5, pp. 3531-3540, 2011.

[27] J. Wang, B. Cao, Q. Chen, and F. Wang, "Combined state of charge estimator for electric vehicle battery pack," Control Engineering Practice, vol. 15, no. 12, pp. 1569-1576, 2007.

[28] K. Ahn, A. A. Trani, and H. Rakha, "Microscopic fuel consumption and emission modeling," in Proceedings of the 78th Annual Meeting of the Transportation Research Board (CDROM), Washington, DC, USA, 1999.

[29] K. Ahn, H. Rakha, A. Trani, and M. van Aerde, "Estimating vehicle fuel consumption and emissions based on instantaneous speed and acceleration levels," Journal of Transportation Engineering, vol. 128, no. 2, pp. 182-190, 2002. 


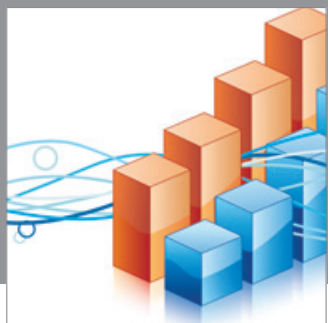

Advances in

Operations Research

mansans

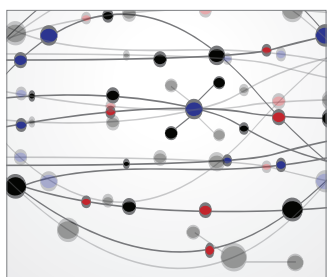

The Scientific World Journal
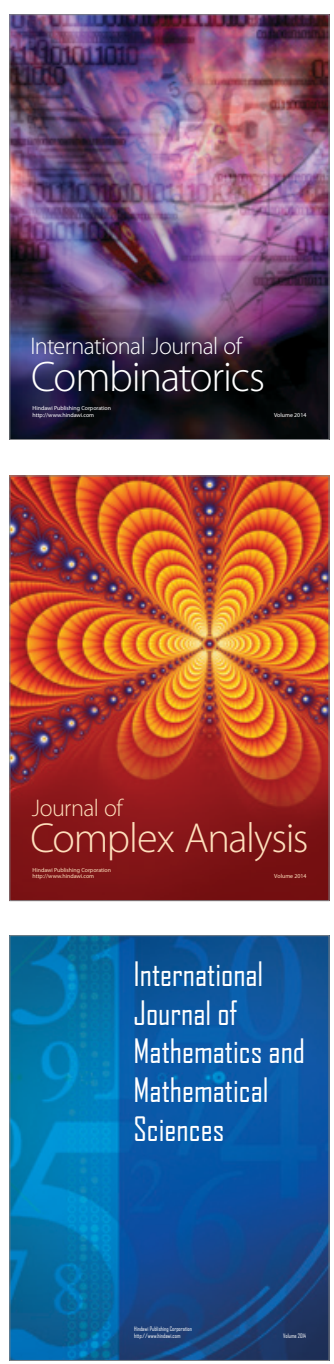
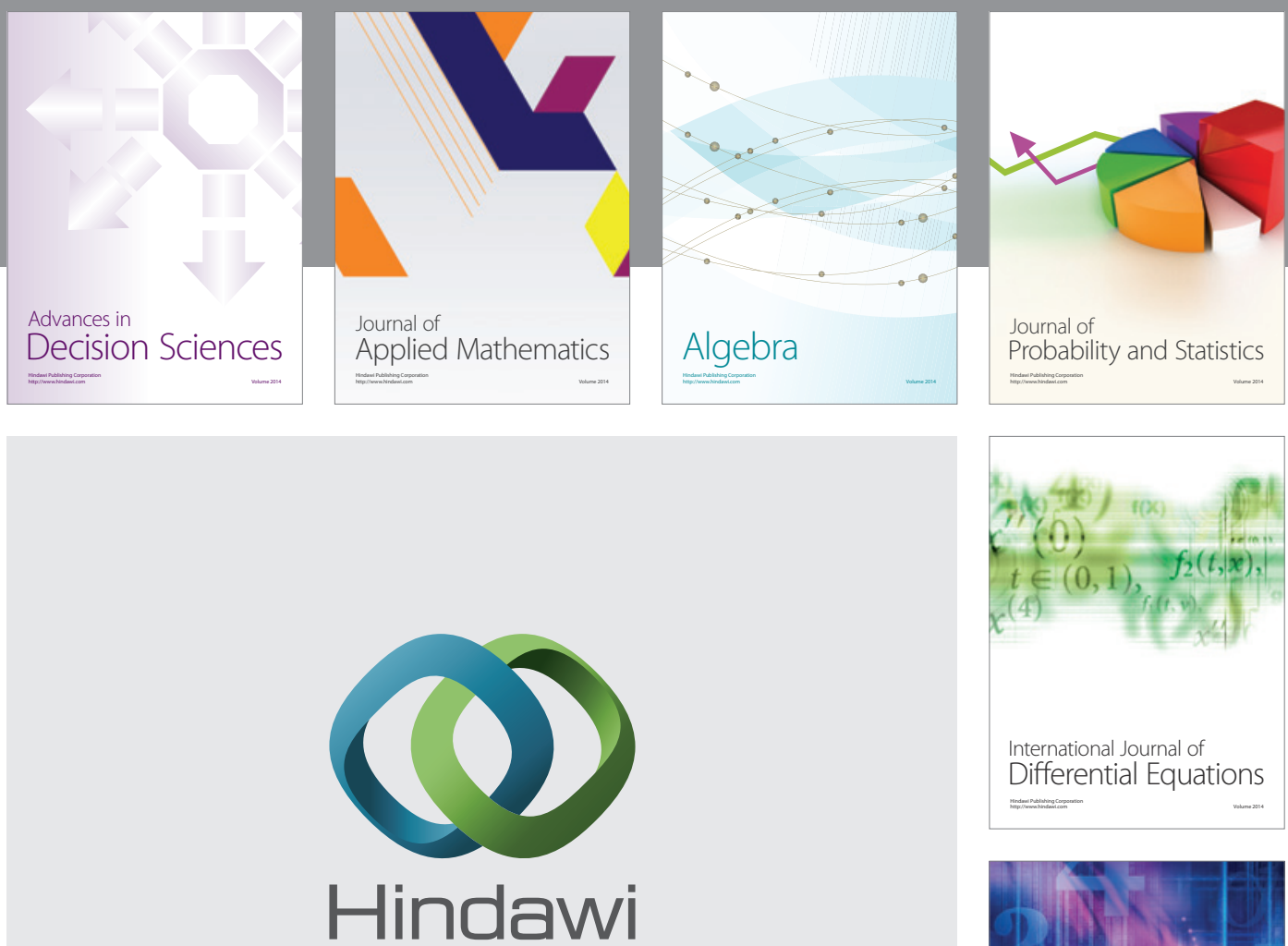

Submit your manuscripts at http://www.hindawi.com
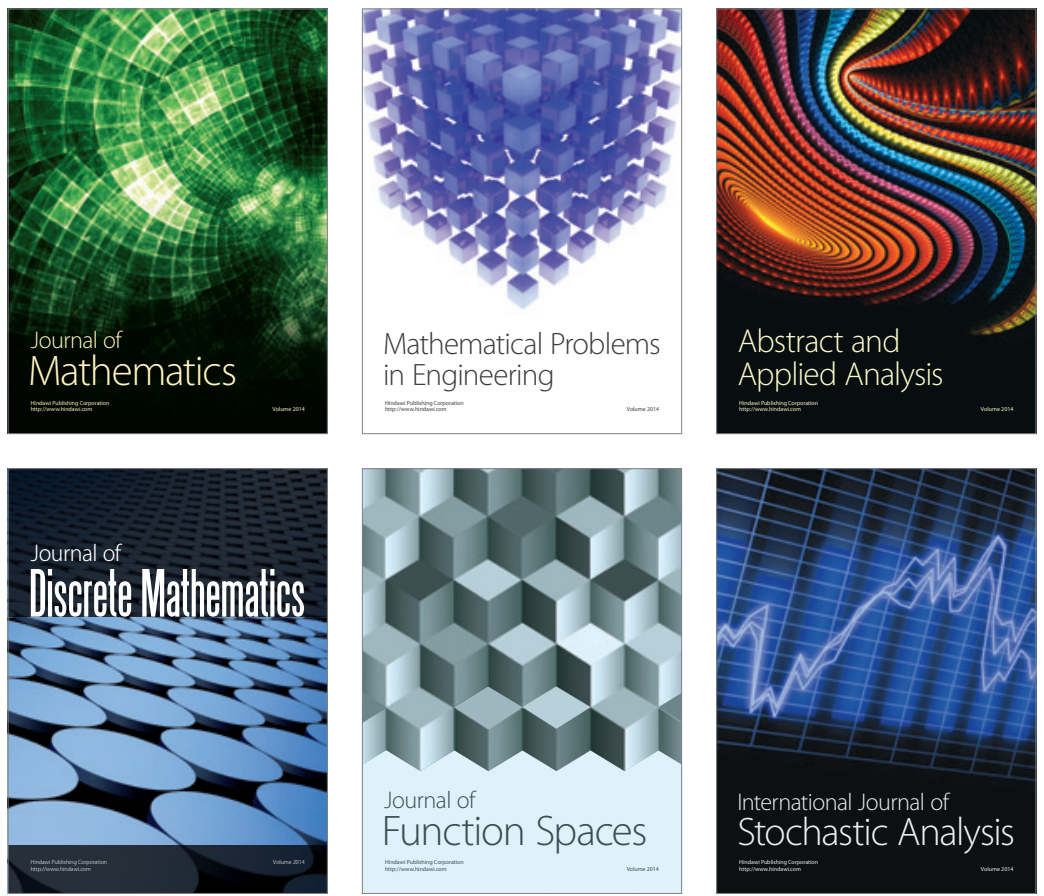

Journal of

Function Spaces

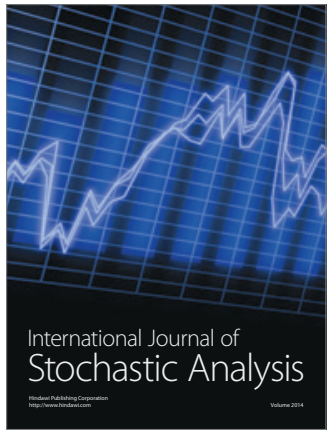

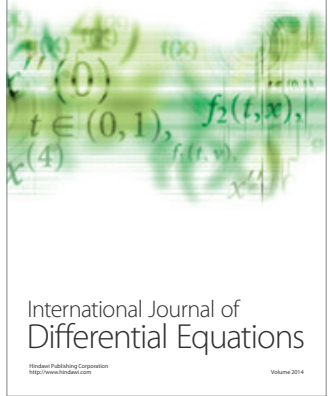
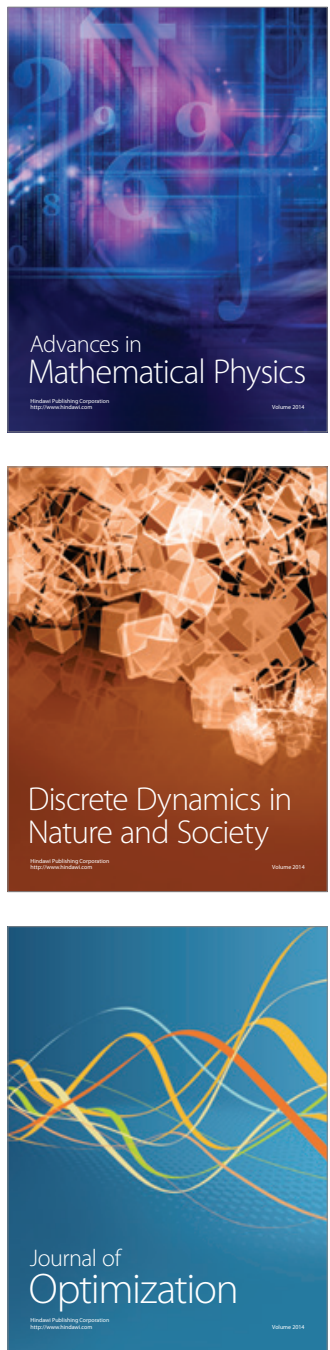